8-1-2014

\title{
Measurements of Turbulent Mixing and Subsiding Shells in Trade Wind Cumuli
}

Jeannine Katzwinkel

Leibniz Institute for Tropospheric Research, jeannine.katzwinkel@tropos.de

Holger Siebert

Leibniz Institute for Tropospheric Research

Thijs Heus

Cleveland State University, t.heus@csuohio.edu

Raymond A. Shaw

Michigan Technological University

Follow this and additional works at: https://engagedscholarship.csuohio.edu/sciphysics_facpub

Part of the Physics Commons

How does access to this work benefit you? Let us know!

Publisher's Statement

(C) Copyright 2014 AMS

\section{Repository Citation}

Katzwinkel, Jeannine; Siebert, Holger; Heus, Thijs; and Shaw, Raymond A., "Measurements of Turbulent Mixing and Subsiding Shells in Trade Wind Cumuli" (2014). Physics Faculty Publications. 221.

https://engagedscholarship.csuohio.edu/sciphysics_facpub/221

This Article is brought to you for free and open access by the Physics Department at EngagedScholarship@CSU. It has been accepted for inclusion in Physics Faculty Publications by an authorized administrator of

EngagedScholarship@CSU. For more information, please contact library.es@csuohio.edu. 


\title{
Measurements of Turbulent Mixing and Subsiding Shells in Trade Wind Cumuli
}

\author{
JEANNINE KATZWINKEL AND HOLGER SIEBERT \\ Leibniz Institute for Tropospheric Research, Leipzig, Germany \\ THIJS Heus \\ University of Cologne, Cologne, Germany \\ RAYMOND A. SHAW \\ Department of Physics, Michigan Technological University, Houghton, Michigan
}

(Manuscript received 22 July 2013, in final form 21 March 2014)

\begin{abstract}
High-resolution measurements of the turbulent, thermodynamic, and microphysical structure of the edges of trade wind cumuli have been performed with the Airborne Cloud Turbulence Observation System. Lateral entrainment of subsaturated air into the cloud region leads to an evaporative cooling effect. The negatively buoyant air partly enhances the compensating downdraft, forming a subsiding shell at cloud edge. Based on the presented observations, the subsiding shell is divided into a turbulent and humid inner shell adjacent to the cloud interior and a nonbuoyant, nonturbulent outer shell. In the trade wind region, continuous development of shallow cumuli over the day allows for an analysis of the properties of both shells as a function of different cloud evolution stages. The shallow cumuli are divided into actively growing, decelerated, and dissolving based on cloud properties. As the cumuli evolve from actively growing to dissolving, the subsaturated environmental air is mixed deeper and deeper into the cloud region and the subsiding shell grows at the expense of the cloud. This measured evolution of the subsiding shell compares favorably with the predictions of a direct numerical simulation of an idealized subsiding shell. The thickness of the measured outer shell decreases with the evolution of the cumuli while the intensity of the downdraft is nearly constant.
\end{abstract}

\section{Introduction}

Cumulus clouds cover large areas of the Earth and play an important role in its energy budget, but their complex dynamical structure is not fully understood. The cloud evolution and its lifetime are influenced by dynamical processes over the full range of the turbulent energy cascade and their interplay among each other. One major issue in this context is the entrainment process at cloud edge whose analysis started with the works of Stommel (1947) and Squires and Warner (1957). Entrainment defines the transport of environmental air into the cloud region either from the side (lateral entrainment) or from above the cloud (cloud-top entrainment). The subsequent mixing between environmental

Corresponding author address: Jeannine Katzwinkel, Leibniz Institute for Tropospheric Research, Permoserstraße 15, 04318 Leipzig, Germany.

E-mail: jeannine.katzwinkel@tropos.de and cloudy air leads to reduction of liquid water content through dilution and the evaporation of cloud droplets, and the resulting latent heating alters the buoyancy field surrounding and inside the cloud. Although the spatial resolution of both model simulation and experiments has improved during the last several decades, there are still open questions about the entrainment process and how it influences cloud microphysics and cloud dynamics.

Here we focus on the small-scale turbulent structure at the edge of cumuli where lateral entrainment and the subsequent mixing between subsaturated environmental and cloudy air leads to the development of a thin "subsiding shell." A first indication of this shell was given by a laboratory experiment performed by Woodward (1959), which indicated a cumulus cloud as a thermal characterized by a body of updrafts surrounded by a shell of downdrafts. This picture was further clarified by aircraft and radar studies of small, maritime cumuli by Jonas (1990) and Knight and Miller (1998). Jonas (1990) 
further pointed out that the presence of the subsiding shell increases the entrainment instability at cloud edge by transporting cloud-top air to lower levels. Tracer experiments performed by Stith (1992) confirm such transport and showed the lateral entrainment of these tracer parcels at lower levels.

The first assumption of Jonas (1990) referred to a mechanical forcing driving the downdrafts, but this was refuted by aircraft observations performed by Rodts et al. (2003). They observed a conspicuous dip in the virtual potential temperature profile and no dip in the total water mixing ratio profile at cloud edge, providing evidence for evaporative cooling as the driving mechanism of the subsiding shell. These observations were compared with a large-eddy simulation (LES) by Heus and Jonker (2008) and the numerical calculation of the individual terms of the vertical momentum equation confirmed the assumption that buoyancy is the dominant force in the subsiding shell. Heus and Jonker (2008) discussed further that the transport of cloud-top air down to lower levels within the subsiding shell refreshes the air at cloud edge, which influences the lateral mixing process and could create a stronger subsiding shell. The same effect can also be generated by an increase in aerosol concentration, which seems to enhance the evaporative cooling rate at cloud edge, leading to enhanced negatively buoyant air at cloud edge (Small et al. 2009). The important role of the subsiding shell for cloud dynamics is further indicated by the increased turbulence intensity within the shell (Siebert et al. 2006b) and the different air properties between subsiding shell and far environment in terms of humidity and buoyancy (Jonker et al. 2008). The entrainment of this preconditioned air instead of subsaturated far-environmental air influences the microphysical response to mixing (Gerber et al. 2008).

A detailed analysis of the shell properties, however, has been challenging because the horizontal extent of a typical shell is comparable to or less than the resolution of LES cloud models or of aircraft data. Two detailed studies of the structure of cumulus clouds are given by Wang et al. (2009) and Wang and Geerts (2010). The first study analyzed the dynamics of the cloud margin with a thickness of $10 \%$ of the cloud diameter, which is characterized by sinking and relatively cold air. The second study characterized negative buoyancy, cold temperature anomalies, and downward motion as typical properties of air in the vicinity of the cloud edge. Nevertheless, they were not able to resolve the small-scale fluctuations due to a spatial resolution of 10 and $5 \mathrm{~m}$, respectively. Also, LESs do not resolve the smallest scales leading mostly to an underestimation of the amplitude of the downdrafts within the subsiding shell by
LESs compared to observations (Heus et al. 2009). Abma et al. (2013) addressed these LES limitations by performing the first fully resolved computational study of the finescale structure of turbulent mixing and the formation of a subsiding shell. The time evolution of a small portion of an idealized subsiding shell was analyzed based on direct numerical simulations (DNS). The results show that during the lateral entrainment and mixing process, the thickness of the subsiding shell grows quadratically and the downdrafts increase linearly with time. Coupling to large scales remains an open aspect of this problem, however.

The current paper presents the first high-resolution measurements of the finescale structure of subsiding shells in cumulus clouds. The measurements were performed with the slow-moving helicopterborne measurement payload Airborne Cloud Turbulence Observation System (ACTOS), which allows for simultaneous and collocated characterization of cloud microphysical, thermodynamic, and turbulence properties at centimeter and decimeter scales. The subsiding shells were measured at the edge of trade wind cumuli. These clouds were selected for this study because the trade wind region is often populated, at any given time, by cumulus convection at all stages of development, which provides the opportunity to analyze the evolution of the subsiding shell. The measurements provide insight into the detailed structure of the subsiding shell and how it varies during the growth and decay stages of cumulus convection. This picture then allows us to make a first effort toward comparing measurements with the idealized DNS results of Abma et al. (2013).

The paper is organized as follows: Section 2 describes the measurement platform ACTOS and its instrumentation. Section 3 describes the basic methods for the data analysis. In section 4 the characteristics of the trade wind cumuli are presented, while section 5 deals with the characteristics of the cloud edge. The comparison of the measurements with DNS is presented in section 6. The paper concludes with a summary and discussion.

\section{Experimental}

High-resolution measurements in trade wind cumuli over the ocean near the eastern coast of Barbados were made during the Clouds, Aerosol, Radiation and Turbulence in the Trade Wind Regime over Barbados (CARRIBA) campaign in November 2010 and April 2011 (Siebert et al. 2013). The measurements were performed with the helicopterborne measurement payload ACTOS, whose main details are summarized here. ACTOS is located below a helicopter on a $140-\mathrm{m}$-long rope and operates with a true airspeed of $20 \mathrm{~m} \mathrm{~s}^{-1}$. Assuming 
a given sampling frequency of $100 \mathrm{~Hz}$, a spatial resolution down to decimeter scale is realized. The turbulence measurements were performed with a hot wire anemometer with a sampling frequency of $2 \mathrm{kHz}$. The influence of droplet impactions on the thin wire under cloudy conditions was considered by applying a despiking algorithm described in Siebert et al. (2007). After despiking, the final resolution is $438 \mathrm{~Hz}$. Besides the turbulence measurements, the following measurements are used for the analysis in this paper: temperature $T$ measured with a special cloud thermometer [ultrafast thermometer (UFT); Haman et al. 1997], absolute humidity $a$ measured with a dewpoint hygrometer (TP 3-S, MeteoLabor, Switzerland) and an open infrared absorption hygrometer (LiCor 7500, LI-COR corporation, United States), pressure $p$ measured with a Barocap Vaisala sensor (Vaisala, Finland), liquid water content (LWC) measured with a particle volume monitor (PVM100A; Gerber et al. 1994), and the three-dimensional wind vector measured with an ultrasonic anemometer Solent HS (Gill Instruments, United Kingdom). The sonic measurements are corrected for attitude and platform velocity. For more information concerning ACTOS the reader is referred to Siebert et al. (2006a). All deployed devices have sufficient resolution for our kind of analysis: the ultrasonic anemometer resolves the wind vector components in centimeters per second (Siebert and Muschinski 2001). The temperature obtained with the UFT shows a resolution of better than $10 \mathrm{mK}$ and the standard deviation due to uncorrelated noise of the PVM$100 \mathrm{~A}$ is about $1 \mathrm{mg} \mathrm{m}^{-3}$ [see Siebert et al. (2003) for a detailed determination of the noise floor for PVM100A and UFT]. All devices have a temporal resolution of at least $100 \mathrm{~Hz}$; therefore, we can safely conclude that our results are not significantly affected by spatial or temporal resolution. All data were collected by performing nearly horizontal flight legs through shallow cumulus clouds approximately $100 \mathrm{~m}$ below cloud top owing to the visual flight restrictions of the helicopter.

\section{Methods}

In this section we outline the basic methods for the analysis of the high-resolution measurements within a large number of trade wind cumuli. All measurements used herein have a spatial resolution of $0.2 \mathrm{~m}$ except the local energy dissipation rate, which is explained later. We identify quantitative criteria for defining the cloud and the subsiding-shell regions within the selected clouds. The selection is based on certain conditions that allow for a statistical analysis. Furthermore, because the trade wind environment allows clouds to be

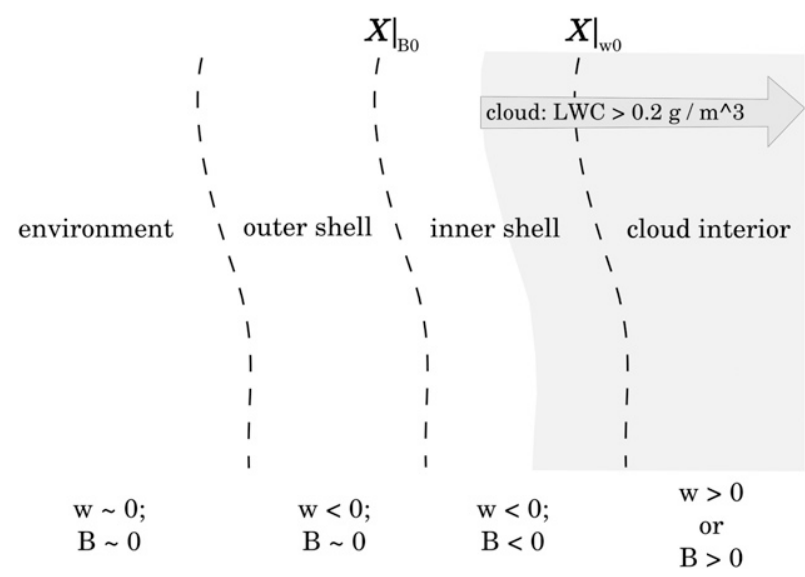

FIG. 1. Illustration of the different cloud and subsiding-shell regions and their characteristics.

sampled at all stages of development, the statistical analysis suggests a natural definition of three evolutionary stages: actively growing, decelerating, and dissolving clouds.

The criteria for the cloud and subsiding shell regions specified here are determined in the context of 1) an effort to be generally consistent with the definitions given in the recent literature on subsiding shells and 2) the fact that ACTOS data are taken at very high resolution and therefore reveal levels of detail in fluctuating quantities that need to be considered in the definitions. The cloud extent is fundamentally ambiguous because of the lack of a clearly defined edge when viewed at high resolution. In past studies (e.g., Rodts et al. 2003) a threshold cloud droplet number density was chosen; here we also use a threshold, but because number density is more difficult to estimate over very short flight lengths, we use liquid water content. The region with LWC $>0.2 \mathrm{~g} \mathrm{~m}^{-3}$ is taken to define the cloud. The length of this region is defined as the cloud diameter. It should be noted that all measurements presented here were taken within about $100 \mathrm{~m}$ of cloud top, so this threshold is relatively conservative. Each flight transect through a cloud is divided into three regions: environment, subsiding shell, and cloud interior. Based on careful observation of the data, and also consistent with the work of Heus and Jonker (2008), the subsiding-shell region is further divided into two regions: one negatively buoyant as a result of cloud mixing and evaporation (called the inner shell) and the other possessing nearly the same buoyancy as the environment but with negative velocity (called the outer shell). The characteristics of these regions are discussed in detail later, but we provide the defining criteria here. A schematic picture of the different regions including the characteristics of the boundaries is shown in Fig. 1. 
The inner shell is characterized by being adjacent to the cloud interior and by being negatively buoyant. It is therefore defined by the following two boundaries: the outside boundary is located where buoyancy $B$ begins to decrease below zero (denoted $\left.X\right|_{B_{0}}$ ), and the inside boundary is located where $w$ changes from downdraft to updraft or where $B$ changes from negative to positive within or at the edge of the region with $\mathrm{LWC}>0.2 \mathrm{~g} \mathrm{~m}^{-3}$ (denoted $\left.X\right|_{w_{0}}$ ).

The outer shell is characterized by not being negatively buoyant like the inner shell, but still showing negative velocities. It is therefore defined by the following two boundaries: the outside boundary is located where $w$ starts to decrease below the environmental value of nearly zero, and the inside boundary is located where $B$ starts to decrease below zero (i.e., the outer boundary of the inner shell).

It should be noted that these criteria allow that the negatively buoyant region of the subsiding shell can encompass a portion of the cloud (i.e., regions with LWC $\left.>0.2 \mathrm{~g} \mathrm{~m}^{-3}\right)$. As opposed to the inner shell, the cloud interior is the part of the cloud that is not directly affected by entrainment of environmental air. The boundary between the inner shell and the cloud interior is denoted with the above mentioned parameter $\left.X\right|_{w_{0}}$.

For the purpose of a statistical analysis of the cloud and subsiding shell regions, we define the following conditions for selecting individual clouds. First, small cloud fragments are excluded from the analysis. This means that the diameter of the cloud and LWC in the cloud region have to be larger than $50 \mathrm{~m}$ and $0.2 \mathrm{~g} \mathrm{~m}^{-3}$, respectively. Second, two neighboring clouds have to be sufficiently well separated from each other so as to define a clear, cloud-free environment between subsequent clouds. Therefore, the distance to the next cloud has to be larger than $100 \mathrm{~m}$. This comparable short distance between two neighboring clouds is critical but a good compromise to get enough clouds for our analysis without observing an interference between to subsequent subsiding shells. With these conditions, 217 clouds are selected.

Typically, in the trade wind region clouds at different stages of evolution can be found close to each other. Frequently, actively growing clouds are observed adjacent to dissolving ones. This is caused by the continuous development of shallow cumuli under quite homogeneous conditions in terms of sea surface temperature and mesoscale dynamics. The observations suggest that the cloud evolution can be divided into three stages of development. Each stage is defined based on the 90th percentile values for updraft velocity and buoyancy, $w_{90 \%}$ and $B_{90 \%}$ respectively, as observed within the cloud (i.e., the region with $\mathrm{LWC}>0.2 \mathrm{~g} \mathrm{~m}^{-3}$ ):
- active, for $w_{90 \%}>0$ and $B_{90 \%}>0$;

- decelerated, for $w_{90 \%}>0$ and $B_{90 \%}<0$; and

- dissolving, for $w_{90 \%}<0$ and $B_{90 \%}<0$.

Further details of the analysis, specific examples, and averaged observations are given later.

The characterization of the subsiding shell regions and the three stages of development is based on buoyancy. Here, we calculate this parameter as $B=$ $g\left(\Theta_{v}-\bar{\Theta}_{v}\right) /\left(\bar{\Theta}_{v}\right)$, where $\Theta_{v}$ is the virtual potential temperature calculated as $\Theta_{v}=\Theta\left(1+0.61 q_{t}-1.61 q_{l}\right)$, where $q_{t}$ and $q_{l}$ denote the total water and liquid water mixing ratios. The overbar denotes the cloud-free environmental mean value calculated for each cloud individually.

The further analysis of the high-resolution measurements is based on the calculation of a local energy dissipation rate $\bar{\varepsilon}_{\tau}$ where we applied two different methods. In the first method, the basic definition of $\bar{\varepsilon}_{\tau}$ is used with the assumption of the validity of the Taylor hypothesis and local isotropy (Wyngaard 2010):

$$
\bar{\varepsilon}_{\tau} \equiv \frac{15 \nu}{\bar{U}_{\mathrm{TAS}}} \overline{\left(\partial_{t} u\right)_{\tau}^{2}},
$$

where $\nu$ denotes the kinematic viscosity, $U_{\text {TAS }}$ and $\partial_{t} u$ denote the true airspeed and the temporal derivative of the longitudinal wind velocity component measured with the hot wire anemometer, and $\overline{(\cdot)_{\tau}}$ denotes the average of the respective parameter over the integration time $\tau$. Here, we apply a moving window with a width of 100 samples to the despiked hot wire data with $438-\mathrm{Hz}$ resolution, which yields a spatial resolution of $\lambda=$ $U_{\text {TAS }} / f \approx 5 \mathrm{~cm}$. This method is conducive to calculating the spatial structure of the $\bar{\varepsilon}_{\tau}$ - especially at cloud edge where high variability exists. The absolute values are underestimated because for an accurate estimate of $\bar{\varepsilon}_{\tau}$, a spatial resolution on the order of $10 \eta \approx 1 \mathrm{~cm}$ is necessary, where $\eta \approx 1 \mathrm{~mm}$ is the Kolmogorov length scale. In the second method, which allows a more precise value of $\bar{\varepsilon}_{\tau}$ to be determined with coarse-grained data, the dissipation rate is estimated from the second-order structure function $S^{(2)}\left(t^{\prime}\right)$ :

$$
\bar{\varepsilon}_{\tau} \simeq \frac{\left\{0.5{\overline{\left[S^{(2)}\left(t^{\prime}\right)\right]}}_{\tau}\right\}^{3 / 2}}{\left(t^{\prime} \bar{U}_{\mathrm{TAS}}\right)},
$$

where $S^{(2)}\left(t^{\prime}\right)=\left[u\left(t+t^{\prime}\right)-u(t)\right]^{2}$. Here, $S^{(2)}$ is estimated for nonoverlapping subrecords of the longitudinal velocity component measured with the ultrasonic anemometer; therefore, no correction for the presence of cloud droplets is necessary. The chosen subrecord length of 100 samples yields a spatial resolution for $\bar{\varepsilon}_{\tau}$ of $20 \mathrm{~m}$. 


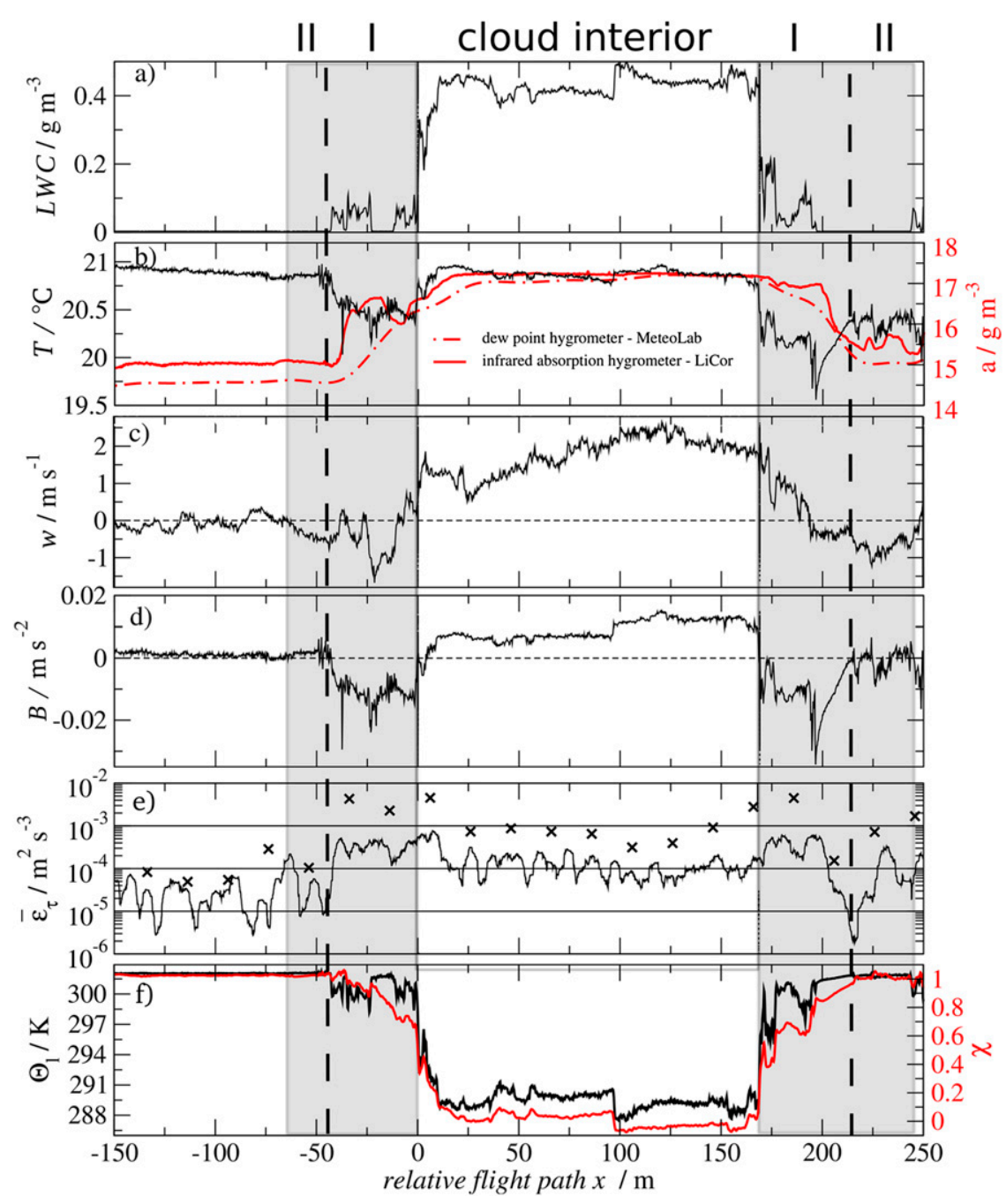

FIG. 2. A single cloud penetration: (a) LWC, (b) temperature and absolute humidity (the red line denotes measurements with the infrared-absorption hygrometer LiCor and the dot-dashed red line marks measurements performed with the dewpoint hygrometer), (c) vertical wind velocity, (d) buoyancy, (e) energy dissipation rate [the black line denotes the direct method and the crosses mark the absolute values calculated based on $\left.S^{(2)}\left(t^{\prime}\right)\right]$, and (f) liquid water potential temperature and mixing fraction. The white region between the two gray regions marks the cloud interior and the two gray regions denote the subsiding shell at the edges of the cloud. The vertical black dashed line divides the subsiding shell into the inner shell (denoted I) and the outer shell (denoted II).

A detailed description of both methods is given in Siebert et al. (2006b).

\section{Statistical cloud analysis}

This section deals with the observed thermodynamic and microphysical properties as well as the turbulent and mean velocity profiles that were used to characterize the cloud interior and the subsiding shell and cloudfree environment regions. In Fig. 2, we illustrate the different cloud regions with a specific example taken from a single cloud penetration on 24 November 2010. For this specific example, the cloud region with LWC > $0.2 \mathrm{~g} \mathrm{~m}^{-3}$ is not directly affected by entrainment of environmental air and so this whole region is equated to the cloud interior. The cloud region is characterized by nearly constant values of $a=17 \mathrm{~g} \mathrm{~m}^{-3}$ and $T=21^{\circ} \mathrm{C}, w>$ 0 with peak values of up to $2.5 \mathrm{~m} \mathrm{~s}^{-1}, B>0$ with an average of about $0.01 \mathrm{~m} \mathrm{~s}^{-2}$, and $\bar{\varepsilon}_{\tau} \sim 10^{-3} \mathrm{~m}^{2} \mathrm{~s}^{-3}$. Hence, this example represents an actively growing cloud. The liquid water potential temperature $\Theta_{l}=\Theta-\left(q_{l} L_{v} \Theta\right) /$ $\left(c_{p} T\right)$, where $L_{v}$ is the latent heat of vaporization and $c_{p}$ is 
the specific heat at constant air pressure (Betts 1973), shows a mean value of $289 \mathrm{~K}$. In the cloud-free environment, $\bar{\varepsilon}_{\tau}$ and $a$ decrease to $10^{-4} \mathrm{~m}^{2} \mathrm{~s}^{-3}$ and $14.5 \mathrm{~g} \mathrm{~m}^{-3}$, respectively, $T$ shows similar values as inside the cloud interior, $w$ and $B$ fluctuate around zero, and $\Theta_{l}=302 \mathrm{~K}$.

The subsiding shell at cloud edge is marked in Fig. 2 by the gray regions located between the cloud-free environment and the cloud interior, adjacent to both sides. For this specific example, these regions are characterized by a residual $\mathrm{LWC}<0.1 \mathrm{~g} \mathrm{~m}^{-3}$ with a high variability, illustrating the eddylike structure of the cloud edge. A closer look shows that inside this subsiding shell region, $\bar{\varepsilon}_{\tau}$ increases from environmental values to values even higher than in the cloud interior (black line in Fig. $2 \mathrm{e})$. At the same point, $B$ starts to decrease. Based on these observations, the subsiding-shell region is further divided into two shells (indicated by the vertical dashed black lines). The inner shell (denoted with "I"), adjacent to the cloud interior, is characterized by reduced values of $T, w<0, B<0$, and $\bar{\varepsilon}_{\tau} \sim 10^{-2} \mathrm{~m}^{2} \mathrm{~s}^{-3}$, again higher compared with the cloud interior. Additionally, $a$ increases from environmental values to nearly cloud values within this region. It should be noted, however, that the humidity measurements do not allow a detailed look at the fluctuations because of the significantly reduced spatial resolution. The dewpoint hygrometer (dotted red line) measures only with a spatial resolution of $20 \mathrm{~m}$ and the LiCor (straight red line) was used as a closed system to reduce the cloud droplet impaction. To analyze the humidity fluctuations in the inner shell, highly resolved measurements such as with a Lymanalpha absorption hygrometer would be necessary. The turbulent and humid inner shell was analyzed with LESs by Heus and Jonker (2008). Between the inner shell and the cloud-free environment the outer shell is observed, here denoted with "II." This region is characterized by temperature and humidity comparable to the environmental values, a slight downdraft with $B \approx 0$, and a comparably small $\bar{\varepsilon}_{\tau}$.

The origin of the air within the inner and outer shell can be investigated by using the liquid water potential temperature and the total water content, both of which are conserved variables during mixing. The outer shell consists mostly of environmental air, which can be seen in Fig. $2 \mathrm{f}$ by the values of $\Theta_{l}$ and mixing fraction $\chi$ (Kain and Fritsch 1990) that are similar to the environment values. Here, $\chi=\left(q_{t}-\bar{q}_{t_{c}}\right) /\left(\bar{q}_{t_{e}}-\bar{q}_{t_{c}}\right)$, where the subscripts $c$ and $e$ denote the cloud and environmental values, respectively, and the overbar denotes the average value in the corresponding regime. In contrast, the air inside the inner shell is a mixture of environmental and cloud air as indicated by the mean value of $\Theta_{l}=$ $299 \mathrm{~K}$ within this inner shell. Also, $\chi$ shows a nearly
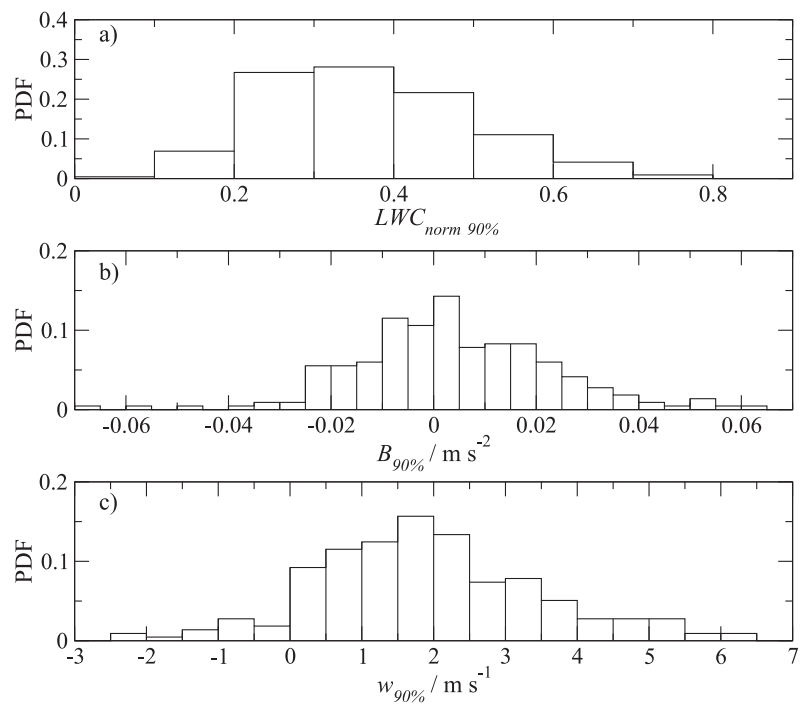

FIG. 3. The variability of all 217 selected clouds is represented by the PDFs of the 90th percentiles of (a) normalized liquid water content, (b) buoyancy, and (c) vertical wind velocity inside the cloud.

linear decrease with same variability toward the cloud interior with a minimum value of 0.7 indicating a mixture of $70 \%$ environmental and $30 \%$ cloudy air. Although, it has been previously assumed that lateral mixing is the leading mechanism for the development of the inner shell (Heus and Jonker 2008), no definite statement about the mixing process can be made based on the data presented here. Evidence for this assumption could be the calculated mixing temperature of $\Theta_{l_{m}}=\chi \Theta_{l_{e}}+(1-\chi) \Theta_{l_{c}}=299.6 \mathrm{~K}$, which is nearly the same as the observed mean value within the shell. But the mean value of $\Theta_{l}$ could also be interpreted as being a result of mixing of cloud air with clear air from above indicated by an estimated lapse rate of $0.5 \mathrm{~K}(100 \mathrm{~m})^{-1}$ for $\Theta_{l}$ under cloud-free conditions. In either case, the turbulent mixing of these two air masses leads to an evaporative cooling effect resulting in negatively buoyant air at cloud edge.

The statistics of the parameters $B_{90 \%}$ and $w_{90 \%}$ which are used to define the different cloud life stages together with the 90th percentile of the normalized LWC (normalized with the calculated adiabatic value) $\left(\mathrm{LWC}_{\mathrm{norm}} 90 \%\right)$ is analyzed. In Fig. 3a, the distribution of $\mathrm{LWC}_{\text {norm }} 90 \%$ varies in a broad range between 0.1 and 0.8 times the adiabaticcalculated value. The probability density function (PDF) of $B_{90 \%}$ shows positive values, indicating actively growing clouds, and also negative values inside the cloud (Fig. 3b). Figure $3 \mathrm{c}$ shows the PDF of $w_{90 \%}$ representing a majority of clouds with an updraft inside the cloud. But a few clouds with downdrafts inside the cloud are measured, indicating dissolving clouds. 


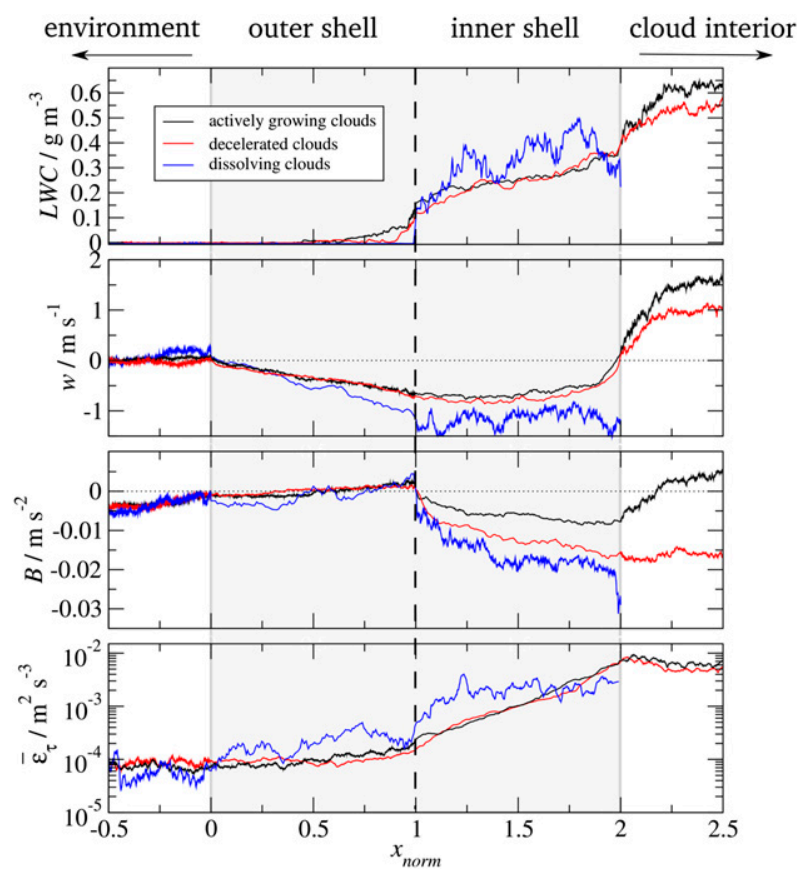

FIG. 4. Rescaled averaged cross sections for (from top to bottom) liquid water content, vertical wind velocity, buoyancy, and energy dissipation rate within the cloud interior and subsiding shellregions. The black curve denotes the actively growing clouds, the red line denotes the decelerated clouds, and the blue line denotes the dissolving clouds. For each cloud individually, the different regions are scaled with their one typical dimension and averaged afterward.

For all three stages of development (actively growing, decelerated, and dissolving), we calculated rescaled averaged cross sections for LWC, $w, B$, and $\bar{\varepsilon}_{\tau}$ within the cloud and subsiding shell regions. The results are shown in Fig. 4, in which the black line represents the average of actively growing clouds, the red line represents the average of the decelerated ones, and the blue line denotes the average of the dissolving clouds. The individual regions are rescaled with their own typical dimension for each cloud individually and are averaged afterward. For all stages, the cloud-free environment is characterized by zero liquid water content, values of $w$ and $B$ around zero, and a small $\bar{\varepsilon}_{\tau}$ on the order of $10^{-4} \mathrm{~m}^{2} \mathrm{~s}^{-3}$. Beside the value of $B$ around zero for all stages, increased variability is obvious within the outer shell. Especially the dissolving clouds differ from the actively growing and decelerated clouds. The dissolving clouds show nearly no LWC and a higher $\bar{\varepsilon}_{\tau}$ within the outer shell. Additionally, the nearly linear decrease of $w$ obvious for all stages is intensified for the dissolving clouds. The characterization of the inner shell with $w<0$ and $B<$ 0 is obvious for all stages of development. The high variability for the dissolving clouds can be explained by the fact that the inner shell covers the whole cloud in this stage of evolution. Hence, in the dissolving case no cloud interior is identified and, therefore, the high values of LWC and $\bar{\varepsilon}_{\tau}$ are found in the inner shell. In the cloud interior, the actively growing and decelerated clouds show only small differences concerning LWC, $w$, and $\bar{\varepsilon}_{\tau}$. The mean buoyancy shows positive values for the actively growing clouds and negative values in the decelerated case, which is in agreement with the definition in section 3 .

\section{Characterization of the cloud edge}

In the following, the analysis of the edges-in particular the subsiding shells (see Fig. 2)—of 217 shallow cumulus clouds is presented. Only edges sampled while entering the cloud have been considered to minimize any possible influence of wetting of the temperature probe.

The characterization of the inner shell is based on three main parameters. One parameter is the thickness of this shell whose calculation based on the definition by Abma et al. (2013):

$$
\delta=\frac{1}{B_{10 \%}} \int_{\left.X\right|_{B_{0}}}^{\left.X\right|_{w_{0}}} B d x,
$$

where $\left.X\right|_{B_{0}}$ and $\left.X\right|_{w_{0}}$ denote the two boundaries of the inner shell. Here, the integral is normalized with the 10th percentile of the buoyancy calculated within the integration limits. The use of the 10th percentile instead of the minimum values as described by Abma et al. (2013) is simply a result of the dependency of the measured minimum value on the measurement resolution. The second and third parameters for characterizing the inner shell are the 10th percentile of buoyancy and the 10th percentile of the vertical wind velocity $w_{10 \%}$ calculated also within the integration limits.

Figure 5 shows examples of cloud edges for each of the cloud evolution stages-the criteria for which were defined in section 3 . These clouds were measured on 14 November 2010 and 14 and 16 April 2011. The $x$ axis shows the distance relative to the entrance of the cloud (CE). For all cases, $200 \mathrm{~m}$ in front of the cloud and $100 \mathrm{~m}$ of the cloud itself are shown. The different regions are marked with vertical black lines and a corresponding identifier (e.g., "env" as environment). The thickness of the inner shell clearly differs in Fig. 5 depending on the stages of evolution. In the case of the actively growing cloud, $\delta$ is about $7 \mathrm{~m}$ with $w_{10 \%}=-1.7 \mathrm{~m} \mathrm{~s}^{-1}$ and $B_{10 \%}=$ $-0.008 \mathrm{~m} \mathrm{~s}^{-2}$. Additionally, the outer shell is apparent with a thickness of $60 \mathrm{~m}$. The decelerated cloud shows a distinct inner shell with a broader thickness of about 


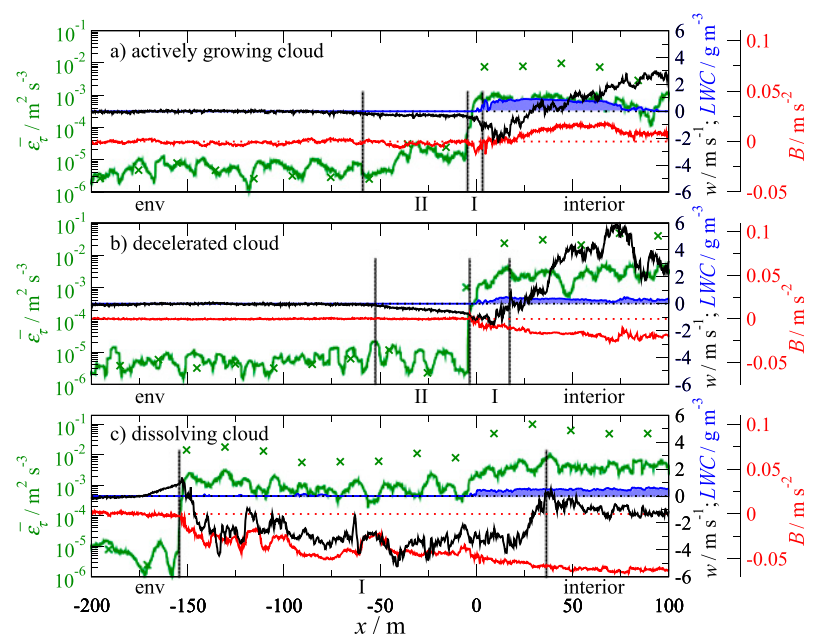

FIG. 5. Examples of cloud edges for each of the three stages of evolution of the trade wind cumuli: (a) actively growing cloud with $w_{90 \%}>0$ and $B_{90 \%}>0$, (b) decelerated cloud with $w_{90 \%}>0$ and $B_{90 \%}<0$, and (c) dissolving cloud with $w_{90 \%}<0$ and $B_{90 \%}<0$. The color code is the same in all panels: blue regions mark the liquid water content, black lines denote the vertical wind velocity, and green and red lines mark the energy dissipation rate and the buoyancy, respectively. The entrance of the cloud interior is denoted by $x=0 \mathrm{~m}$, indicated by LWC $>0.2 \mathrm{~g} \mathrm{~m}^{-3}$.

$20 \mathrm{~m}$ and a downdraft of $w_{10 \%}=-1.5 \mathrm{~m} \mathrm{~s}^{-1}$ and $B_{10 \%}=$ $-0.01 \mathrm{~m} \mathrm{~s}^{-2}$. Additionally, a smaller thickness of $45 \mathrm{~m}$ is observed for the outer shell. In the case of the dissolving cloud an intense downdraft inside the inner subsiding shell of $w_{10 \%}=-3.8 \mathrm{~m} \mathrm{~s}^{-1}$ and $B_{10 \%}=-0.06 \mathrm{~m} \mathrm{~s}^{-2}$ is observed. The thickness of this shell increases to nearly $170 \mathrm{~m}$, and no outer shell is observed.

Figure 6 shows the PDFs of the characteristic parameters $\left(w_{10 \%}, B_{10 \%}\right.$, and $\left.\delta\right)$ of the inner shells. The measured 10th percentile of downdraft inside the inner shells varies between 0 and $-5 \mathrm{~m} \mathrm{~s}^{-1}$ (Fig. 6a). Figure 6b shows the PDF of $B_{10 \%}$, where most of the values lie in the range from 0 to $-0.02 \mathrm{~m} \mathrm{~s}^{-2}$. Additionally, clouds with a positive buoyancy are observed and also a few clouds show a very strong negative buoyancy as low as $-0.08 \mathrm{~m} \mathrm{~s}^{-2}$. The PDF of $\delta$ is shown in Fig. $6 \mathrm{c}$, where the $y$ axis is logarithmic for better clarity. The values vary from below 1 to $175 \mathrm{~m}$ with only a few values observed with $\delta>80 \mathrm{~m}$.

Table 1 summarizes the median properties of the cloud and subsiding-shell regions as a function of the evolution stages. All parameters were first calculated for individual clouds and afterward averaged giving one median value for each evolution stage. The number of clouds grouped in the different evolution stages is given by $n$. The majority of the observed clouds were actively growing; thus, only a few dissolving clouds are considered. This is a result of the stringent criteria for cloud
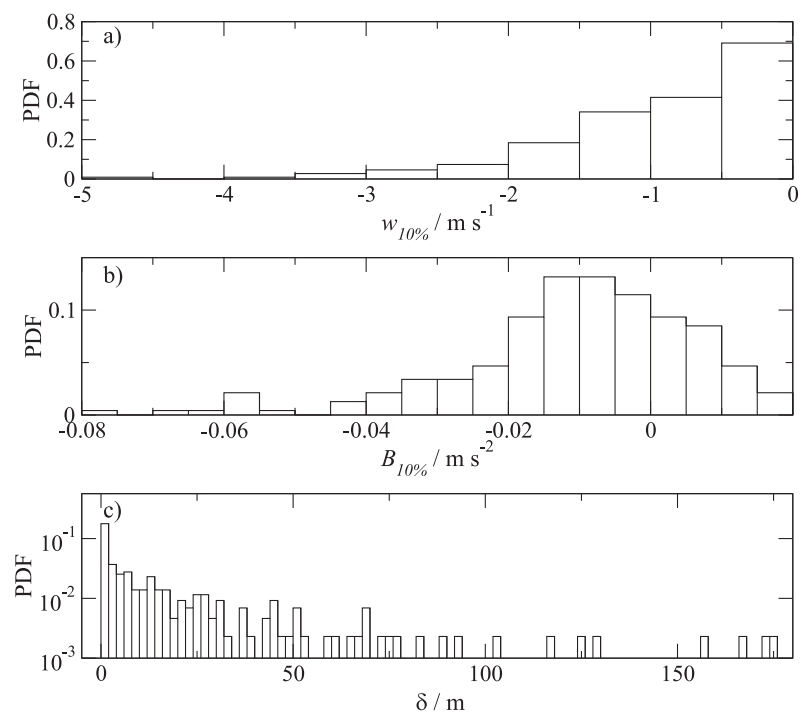

FIG. 6. PDF of (a) minimum downdraft velocity, (b) minimum buoyancy inside the inner shell, and (c) the thickness of the inner shell.

selection. Most of the dissolving clouds are rejected because of their low values of LWC or the narrow cloud diameter. With the evolution from an actively growing cloud to a dissolving one, $w_{90 \%}$ decreases from $2.2 \mathrm{~m} \mathrm{~s}^{-1}$ updraft to a downdraft of $-0.8 \mathrm{~m} \mathrm{~s}^{-1}$, while within the inner shell, an increase in the absolute values of $w_{10 \%}$ and $B_{10 \%}$ is observed. Furthermore, $\left.X\right|_{w_{0}}$ shifts more and more into the cloud region, which is indicated by an increasing distance between CE and $\left.X\right|_{w_{0}}\left[\Delta\left(\left.X\right|_{w_{0}}-\mathrm{CE}\right)\right]$. The mixing process and evaporation of cloud droplets therefore leads to an increasing thickness of the inner shell $\delta$, while the cloud diameter decreases. A nearly constant distance between $\left.X\right|_{B_{0}}$ of the inner shell and the middle of the cloud region (CM) at around $90 \mathrm{~m}$ $\left[\Delta\left(\mathrm{CM}-\left.X\right|_{B_{0}}\right)\right]$ supports the results of Abma et al. (2013) that the inner shell expands primarily into the cloud region. The outer shell is observed at the edge of actively growing clouds with a relative frequency of $82 \%$, but also half of the dissolving clouds are surrounded by such a shell. With the evolution from an actively growing cloud to a dissolving one, the median thickness of the outer shell $\delta_{d}$ decreases, while the downdraft inside this shell fluctuates around $w_{d} \sim$ $-0.3 \mathrm{~m} \mathrm{~s}^{-1}$ for all stages of evolution.

\section{Comparison with model results}

In this section, we explore the consistency of our statistical results with the direct numerical simulations by Abma et al. (2013). A brief description of the model is given here; for further details, the reader is referred to 
TABLE 1. Median properties of all selected clouds for each stage of evolution. These properties are the 90th percentile of $w$ inside the cloud $w_{90 \%}, 10$ th percentile of the downdraft velocity $w_{10 \%}$ and the buoyancy $B_{10 \%}$ inside the inner shell, distance between the boundary of the inner shell close to the cloud region $\left.X\right|_{w_{0}}$ and CE $\left[\Delta\left(\left.X\right|_{w_{0}}-\mathrm{CE}\right)\right]$, the thickness of the inner shell $\delta$, cloud diameter (cd), distance between the cloud-free boundary of the inner shell $\left.X\right|_{B_{0}}$ and the middle of the cloud $(\mathrm{CM})\left[\Delta\left(\mathrm{CM}-\left.X\right|_{B_{0}}\right)\right]$, the relative frequency of the outer shell $f$, median thickness of the outer shell $\delta_{d}$, and median downdraft velocity $w_{d}$ inside the outer shell.

\begin{tabular}{lccc}
\hline \hline & $\begin{array}{c}\text { Actively growing } \\
\left(w_{90 \%}>0, B_{90 \%}>0\right)\end{array}$ & $\begin{array}{c}\text { Decelerated } \\
\left(w_{90 \%}>0, B_{90 \%}<0\right)\end{array}$ & $\begin{array}{c}\text { Dissolving } \\
\left(w_{90 \%}<0, B_{90 \%}<0\right)\end{array}$ \\
\hline$n$ & 122 & 79 & 14 \\
$w_{90 \%}\left(\mathrm{~m} \mathrm{~s}^{-1}\right)$ & 2.2 & 1.6 & -0.8 \\
$w_{10 \%}\left(\mathrm{~m} \mathrm{~s}^{-1}\right)$ & -0.3 & -1.0 & -1.9 \\
$B_{10 \%}\left(\mathrm{~m} \mathrm{~s}^{-2}\right)$ & -0.002 & -0.016 & -0.033 \\
$\Delta\left(\left.X\right|_{w_{0}}-\mathrm{CE}\right)(\mathrm{m})$ & 6 & 12 & 76 \\
$\delta(\mathrm{m})$ & 2 & 16 & 82 \\
$\mathrm{~cd}(\mathrm{~m})$ & 178 & 120 & 82 \\
$\Delta\left(\mathrm{CM}-\left.X\right|_{B_{0}}\right)(\mathrm{m})$ & 99 & 82 & 96 \\
$f(\%)$ & 82 & 70 & 45 \\
$\delta_{d}(\mathrm{~m})$ & 68 & 47 & 6 \\
$w_{d}\left(\mathrm{~m} \mathrm{~s}^{-1}\right)$ & -0.32 & -0.25 & -0.45 \\
\hline
\end{tabular}

Abma et al. (2013). DNS fully resolves turbulence down to dissipation scales, sacrificing instead the large-eddy structure through an idealized setup. Furthermore, approximations are made in the treatment of cloud microphysics. To simulate the lateral entrainment process at the side of a single shallow cumulus, the model is based on a two-layer system including an environmental and a cloud region that are well separated. Both regions are characterized by zero vertical wind velocity and zero buoyancy. Therefore, in contrast to our measurements, no compensation flow exists at cloud edge. A broadband perturbation initiates the mixing between the subsaturated environmental air and the cloudy air. The subsequent evaporation of droplets leads to a cooling effect, resulting in negatively buoyant air at cloud edge. This initiates the development of the buoyant subsiding shell. With increasing time, the simulated subsiding shell grows owing to further turbulent mixing between the environmental and cloudy air. The DNS results basically show a quadratic growth in the thickness of the subsiding shell and a linear increase of the downdraft velocity with time. Based on self-similarity arguments, these findings can be described by the following equations (Abma et al. 2013):

$$
\begin{aligned}
\delta_{m}(t)= & \left(c_{1} c_{2} \frac{B_{m}}{2}\right)\left(t-t_{m 1}\right)^{2} \\
& +\sqrt{2 c_{1} c_{2} B_{m} \delta_{m 1}}\left(t-t_{m 1}\right)+\delta_{m 1}, \\
w_{m}= & \sqrt{2 \frac{c_{1}}{c_{2}} B_{m} \delta_{m}} .
\end{aligned}
$$

Here, $\delta_{m}$ is the thickness of the simulated subsiding shell and $B_{m}$ and $w_{m}$ denote the minimum values of buoyancy and vertical wind velocity within the simulated subsiding shell. According to the simulation of Abma et al. (2013), the initial condition $t_{m 1}$ and $\delta_{m 1}$ are set to $5 \mathrm{~s}$ and $0.054 \mathrm{~m}$, respectively, and the constants $c_{1}$ and $c_{2}$ are determined to be 0.24 and 0.1 .

The basis for the comparison of measurements and the simulation is given by the main properties of the inner shell: $\delta, w_{10 \%}$, and $B_{10 \%}$ for measurements and $\delta_{m}$, $w_{m}$, and $B_{m}$ for the simulated subsiding shell. While the measurements are performed at the edges of numerous clouds in different stages of evolution, the simulation considers one single cloud edge and the evolution of the corresponding subsiding shell. This evolution is calculated every $10 \mathrm{~s}$ according to Eqs. (4) and (5), and it was observed that the value of $B_{m}$ increases linearly until reaching a typical value of $0.02 \mathrm{~m} \mathrm{~s}^{-2}$ during the first $30 \mathrm{~s}$ and remains constant afterward. Consistent with the latter stages of the simulation, $B_{m}$ is taken as a constant for the subsequent comparison with data.

Figure 7 shows the correlation between downdraft intensity and thickness of the buoyant subsiding shell in Fig. 7a and between downdraft intensity and buoyancy in Fig. 7b. All correlations are plotted for the simulated subsiding shell (red lines) and for the measured values of the inner shell (boxes). In Fig. 7a, the simulation shows a quadratic dependence of $\delta_{m}$ on $w_{m}$. The measured $\delta$ also increases with decreasing $w_{10 \%}$, but the measurement uncertainty does not allow for definitive statement about a linear, quadratic, or other relationship. The decreasing number of measured values with increasing downdraft intensity leads to an increasing statistical uncertainty that makes rigorous conclusions difficult. Nevertheless, the measurements are in the range of the model prediction and a similar tendency and absolute values are observed. A similar behavior is seen for the correlations shown in Fig. 7b. After the first seconds, the 
a)

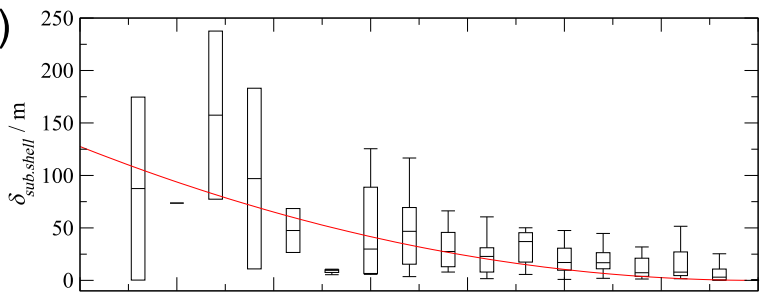

b)

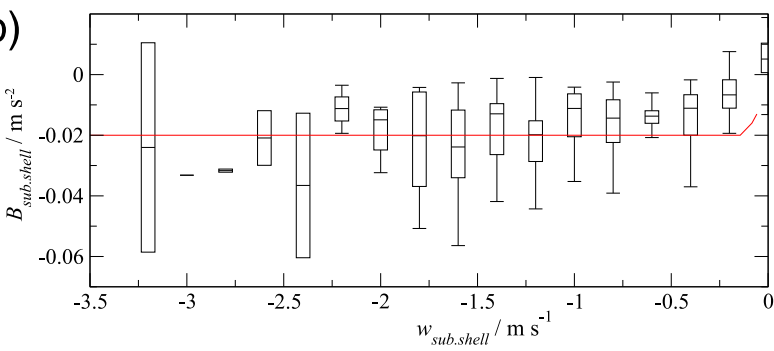

FIG. 7. Comparison of the measured $\left(\delta, w_{10}, B_{10 \%}\right.$; boxes $)$ and simulated $\left(\delta_{m}, w_{m}, B_{m}\right.$; red lines) properties of the inner shell: (a) thickness of the inner shell vs the downdraft velocity in the inner shell and (b) buoyancy vs the downdraft velocity in the inner shell.

simulated value $B_{m}$ remains constant with increasing downdraft intensity. The same tendency is evident in the measurements despite their relatively large spread.

\section{Summary and discussion}

Helicopterborne observations with decimeter resolution were performed during the CARRIBA campaign over Barbados. The observations provide a detailed insight into the edges of trade wind cumuli. The cloud edges were characterized by highly increased turbulence compared to the cloud-free environment but also compared to the cloud region, which is an indication for strong mixing. The key finding was a well-pronounced subsiding shell that was observed in all of the 217 analyzed clouds. The width and the downdraft velocity of these shells were found to depend on the evolution stage of the clouds, which was defined by cloud parameters such as buoyancy and updraft velocity. The findings are illustrated in Fig. 8 for three typical cloud evolution stages defined as an actively growing cloud defined by positive buoyancy resulting in updrafts $(B>0$ and $w>$ 0 ) in the cloud (illustrated by the blue line) (Fig. 8a), a decelerated cloud type with negative buoyancy $(B<0)$ but still updrafts with reduced magnitude $(w>0)$ (Fig. $8 b$ ), and a dissolving cloud with negative buoyancy resulting in downdrafts $(B<0$ and $w<0)$ (Fig. 8c). Because of flight restrictions, the helicopter had to remain above the clouds; therefore, all measurements were performed within $100 \mathrm{~m}$ of cloud top as illustrated by the red horizontal line (see Fig. 8). The measured cloud diameter $(80-100 \mathrm{~m})$ is probably smaller compared to a typical cloud diameter as observed in the middle part of a cloud, but it is expected that the general results can be extended also to lower regions of the cloud.

The measured actively growing clouds (Fig. 8a) are characterized by a comparably broad cloud region (cd) with strong updrafts and are surrounded by a thin subsiding shell. The light gray region denotes the nonbuoyant, nonturbulent outer shell and the dark gray region marks the turbulent and buoyant inner shell, which has been developed because of the entrainment and mixing process. Both regions are characterized by

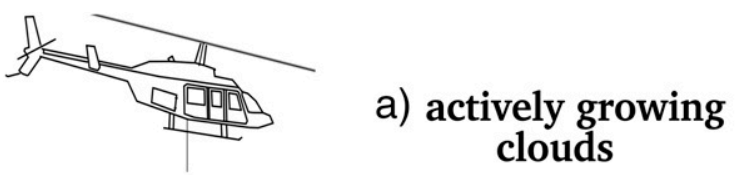

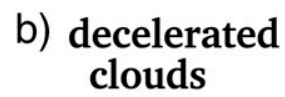
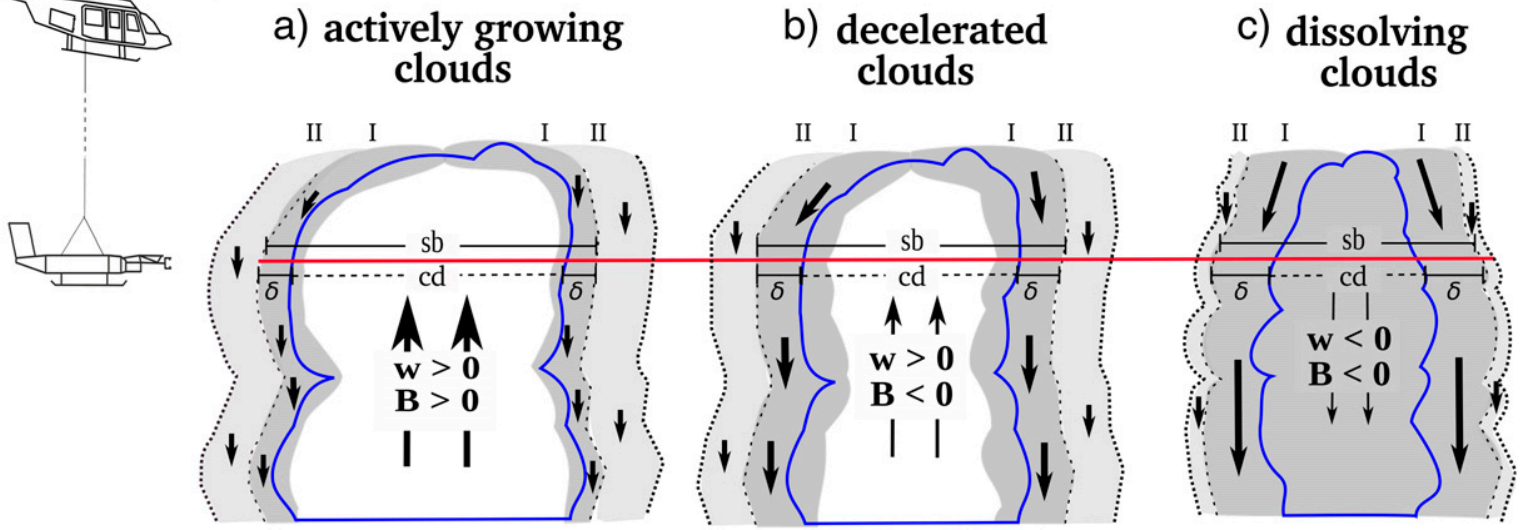

FIG. 8. Schematic picture of the evolution of the cloud (blue line) and the subsiding shell regions as a function of the three stages of evolution [(a) actively growing, (b) decelerated, and (c) dissolving]. The light gray region indicates the outer shell, the dark gray region indicates the inner shell, and the white region within the cloud denotes the cloud interior. Here, $\delta$ marks the distance from beginning of the inner shell to the entrance of the cloud interior, the thickness of the cloud is marked with cd, and sb marks the thickness of the whole system. 
a weak downdraft while the outer shell is typically broader than the thickness $\delta$ of the inner shell. In this case, only a small portion of the cloud is influenced by entrainment of environmental air and so a broad cloud interior (illustrated by the white region within the cloud) is observed. With the evolution to decelerated clouds (Fig. 8b), the subsaturated environmental air is mixed deeper into the cloud and the inner shell grows at the expense of the cloud interior. The width of the entire cloud, including cloud and subsiding-shell regions, was observed to remain nearly constant. Evaporative cooling due to lateral entrainment enhances the downdrafts inside the inner shell, while the updrafts inside the cloud region are decelerated because of negative buoyancy. In contrast to the inner shell, the thickness of the outer shell is reduced while the downdrafts in this region remain nearly constant. In the dissolving stage of the cloud lifetime (Fig. 8c), the width and the downdraft velocity of the inner shell increase further until the inner shell covers the whole cloud. Hence, no cloud interior is observed and the whole cloud is influenced by the entrainment of environmental air, which may lead to the complete dissolving of the cloud. Dissolving clouds show a very small outer shell with a downdraft intensity that is similar to the other two stages. The increasing thickness of the inner shell with cloud evolution indicates an increasing entrainment of preconditioned air instead of subsaturated far-environmental air influencing the microphysical cloud properties (Gerber et al. 2008).

The qualitative agreement of the observed correlations between downdraft intensity, width, and buoyancy of the inner shell with results gained from DNS by Abma et al. (2013) help corroborate the existence of a functional relationship between shell properties and cloud evolution stage. The time evolution of $\delta_{m}$ in the DNS [cf. Eq. (4)] allows an estimate of the evolution time of the measured inner shell. For example, if we consider an observed downdraft velocity of $w_{10 \%}=-3.2 \mathrm{~m} \mathrm{~s}^{-1}$ and the corresponding inner-shell thickness of $\delta=100 \mathrm{~m}$ (cf. Fig. 7a), with the help of Eqs. (4) and (5), this data point can be related to a model evolution time of $t=670 \mathrm{~s}$. But one must keep in mind that this model evolution time starts after the duration of the spinup phase and so does not represent a real cloud age. Additionally, the model ignores any large-scale cloud dynamics, making an interpretation of this time even more difficult. However, this rough estimate provides some idea for future research as to how to estimate lifetime of an observed cloud as a function of shell width.

Our observations of the subsiding shell are also in good agreement with results derived from LES of an unsaturated convective mixed region around a liquid water cloud (Zhao and Austin 2005a,b). The dimensions of their modeled trade wind cumuli are comparable to our observations, which allows a direct comparison. The unsaturated convective mixed region in the LES is first smaller than the liquid water cloud and grows in thickness with the evolution of the cloud. It contains roughly half of the liquid water cloud volume when the cloud top reaches its maximum height. This is of the same magnitude as the relation between our measured cloud and the subsiding shell thickness. The unsaturated convective mixed region reaches its maximum thickness shortly before the liquid water cloud is completely evaporated.

In the case of an actively growing cloud, the airflow inside the outer shell might be explained as a compensating flow owing to the strong updrafts inside the cloud region. This picture is supported by the analysis of Heus and Jonker (2008), who pointed out that $10 \%$ of the upward mass flux in the cloud is compensated in the region with $B<0$ while another $13 \%$ is compensated by being dragged along downward with the buoyant subsiding shell. A more detailed analysis of the massflux compensation by Jonker et al. (2008) leads to the conclusion that "the compensating downward mass flux takes place in the immediate proximity of clouds and not in the form of a weak uniformly subsiding motion."

The whole subsiding shell, including the outer shell, is also observed in the stage of dissolving clouds, so it follows that there must be a mechanism that still results in downward motion, although the upward mass flux in the cloud is reduced. The details of the driving forces of the outer shell are not fully understood yet, but the observations provide the basis for some further speculations. One possibility for the driving force of the outer flow is that the air adjacent to the inner shell could be dragged along with the downdrafts inside the inner shell through lateral turbulent transport of vertical momentum. Another idea is based on observations by Abma et al. (2013), who showed that the minima of the buoyancy and of the vertical velocity fields in the buoyant subsiding shell move toward the cloud interior with different speed: the buoyancy minimum moves faster toward the cloud interior compared with the velocity minimum. However, neither explanation is easily reconciled with the observation that the outer shell is nearly nonturbulent. Finally, one might also speculate that the outer shell could be the very diluted remnants of a previous inner shell that is so diluted that the properties are close to the environment and the turbulent kinetic energy has almost dissipated, but in which the air is still subsiding because of inertia. The current measurements do not necessarily favor any of these possible mechanisms, so further investigations of the outer shell are needed. A starting point would be to perform similar measurements but at different cloud heights. 
We have presented measurements of the finescale properties of cumulus cloud edges, obtained with the helicopterborne measurement platform ACTOS. A statistical analysis of 217 clouds leads to a detailed picture of the turbulent, thermodynamic, and microphysical structure of the subsiding-shell region including its changes during cloud evolution from actively growing to decelerating and finally to dissolving. An analysis of conserved variables and the velocity and buoyancy fields confirms that the subsiding shells are a result of mixing between environmental and cloud air. The present observations indicate that clouds are influenced unequally by the entrainment of environmental air depending on their stage of development. This is caused by different properties of the entrained air due to the increasing thickness of the inner shell. Furthermore, these shells are often characterized by higher turbulent energy dissipation rates than in the cloud. An initial effort toward comparing measurements to the idealized development of a cloud-edge mixing region in a direct numerical simulation gave some general agreement-most notably that shell thickness increases monotonically as downdrafts become more intense. The measurements further suggest that the inner-shell region grows at the expense of the cloud interior, as illustrated in Fig. 8. Many questions remain, among which are the origin of entrained air (lateral versus from cloud top) and the mechanism driving downward velocity in the observed outer shells. Measurements under a wider range of environmental conditions and at a larger range of heights as well as computational studies bridging DNS and LES scales will provide more insight. Finally, the influence of lateral entrainment and subsiding shell formation on the evolution of cloud microphysical properties remains an open problem.

Acknowledgments. We thank the two pilots Alwin Vollmer and Milos Kapetanovic for the great helicopter flights. Thanks also to Paul Archer and to National Helicopters in Canada for organizing and providing the helicopter service. Many thanks also to the team from the Barbados Concorde Experience who hosted the ACTOS and helicopter team. We are grateful to Christoph Klaus and Dieter Schell from the enviscope company for their excellent technical support during the campaigns and acknowledge David Farrell and Damien Prescod from CIMH for their support in terms of local logistics. We gratefully acknowledge Deutsche Forschungsgemeinschaft (DFG) for funding this project within the priority program Metström (SI 1534/2-2) and the DFG-project (SI 1534/3-1). Raymond A. Shaw's participation in this work was supported by NSF Grant AGS-1026123.

\section{REFERENCES}

Abma, D., T. Heus, and J. P. Mellado, 2013: Direct numerical simulation of evaporative cooling at the lateral boundary of shallow cumulus clouds. J. Atmos. Sci., 70, 2088-2102, doi:10.1175/ JAS-D-12-0230.1.

Betts, A., 1973: Non-precipitating cumulus convection and its parameterization. Quart. J. Roy. Meteor. Soc., 99, 178-196, doi:10.1002/qj.49709941915.

Gerber, H., B. G. Arends, and A. S. Ackerman, 1994: New microphysics sensor for aircraft use. Atmos. Res., 31, 235-252, doi:10.1016/0169-8095(94)90001-9.

— G. M. Frick, J. B. Jensen, and J. G. Hudson, 2008: Entrainment, mixing, and microphysics in trade-wind cumulus. J. Meteor. Soc. Japan, 86A, 87-106, doi:10.2151/jmsj.86A.87.

Haman, K. E., A. Makulski, S. P. Malinowski, and R. Busen, 1997: A new ultrafast thermometer for airborne measurements in clouds. J. Atmos. Oceanic Technol., 14, 217-227, doi:10.1175/ 1520-0426(1997)014<0217:ANUTFA > 2.0.CO;2.

Heus, T., and H. J. J. Jonker, 2008: Subsiding shells around shallow cumulus clouds. J. Atmos. Sci., 65, 1003-1018, doi:10.1175/ 2007JAS2322.1.

— J. Pols, C. Freek, J. Jonker, J. Harm, A. V. den Akker, E. Harry, and D. H. Lenschow, 2009: Observational validation of the compensating mass flux through the shell around cumulus clouds. Quart. J. Roy. Meteor. Soc., 135, 101-112, doi:10.1002/qj.358.

Jonas, P. R., 1990: Observations of cumulus cloud entrainment. Atmos. Res., 25, 105-127, doi:10.1016/0169-8095(90)90008-Z.

Jonker, H. J. J., T. Heus, and P. P. Sullivan, 2008: A refined view of vertical mass transport by cumulus convection. Geophys. Res. Lett., 35, L07810, doi:10.1029/2007GL032606.

Kain, J. S., and J. M. Fritsch, 1990: A one-dimensional entraining/ detraining plume model and its application in convective parameterization. J. Atmos. Sci., 47, 2784-2802, doi:10.1175/ 1520-0469(1990)047<2784:AODEPM>2.0.CO;2.

Knight, C. A., and L. J. Miller, 1998: Early radar echoes from small, warm cumulus: Bragg and hydrometeor scattering. J. Atmos. Sci., 55, 2974-2992, doi:10.1175/1520-0469(1998)055<2974: EREFSW $>2.0 . \mathrm{CO} ; 2$

Rodts, S. M. A., P. G. Dnynkerke, and H. J. J. Jonker, 2003: Size distributions and dynamical properties of shallow cumulus clouds from aircraft observations and satellite data. J. Atmos. Sci., 60, 1895-1912, doi:10.1175/1520-0469(2003)060<1895: $\mathrm{SDADPO}>2.0 . \mathrm{CO} ; 2$.

Siebert, H., and A. Muschinski, 2001: Relevance of a tuning-fork effect for temperature measurements with the Gill Solent HS ultrasonic anemometer-thermometer. J. Atmos. Oceanic Technol., 18, 1367-1376, doi:10.1175/1520-0426(2001)018<1367: ROATFE $>2.0 . \mathrm{CO} ; 2$.

, M. Wendisch, T. Conrath, U. Teichmann, and J. Heintzenberg, 2003: A new tethered balloon-borne payload for fine-scale observations in the cloudy boundary layer. Bound.-Layer Meteor., 106, 461-482, doi:10.1023/A:1021242305810.

, H. Franke, K. Lehmann, R. Maser, E. W. Saw, D. Schell, R. A. Shaw, and M. Wendisch, 2006a: Probing fine-scale dynamics and microphysics of clouds with helicopter-borne measurements. Bull. Amer. Meteor. Soc., 87, 1727-1738, doi:10.1175/BAMS-87-12-1727.

, K. Lehmann, and M. Wendisch, 2006b: Observations of small scale turbulence and energy dissipation rates in the cloudy boundary layer. J. Atmos. Sci., 63, 1451-1466, doi:10.1175/ JAS3687.1. 
- - - and R. A. Shaw, 2007: On the use of hot-wire anemometers for turbulence measurements in clouds. J. Atmos. Oceanic Technol., 24, 980-993, doi:10.1175/JTECH2018.1.

— , and Coauthors, 2013: The fine-scale structure of the trade wind cumuli over barbados-An introduction to the CARRIBA project. Atmos. Chem. Phys., 13, 10 061-10 077, doi:10.5194/ acp-13-10061-2013.

Small, J. D., P. Y. Chuang, G. Feingold, and H. Jiang, 2009: Can aerosol decrease cloud lifetime? Geophys. Res. Lett., 36, L16806, doi:10.1029/2009GL038888.

Squires, P., and J. Warner, 1957: Some measurement in the orographic cloud of the island of Hawaii and of trade wind cumuli. Tellus, 9, 475-494, doi:10.1111/j.2153-3490.1957.tb01909.x.

Stith, J. L., 1992: Observations of cloud-top entrainment in cumuli. J. Atmos. Sci., 49, 1334-1347, doi:10.1175/ 1520-0469(1992)049<1334:OOCTEI >2.0.CO;2.

Stommel, H., 1947: Entrainment of air into a cumulus cloud. J. Meteor., 4, 91-94, doi:10.1175/1520-0469(1947)004<0091:EOAIAC >2.0.CO;2.
Wang, Y., and B. Geerts, 2010: Humidity variations across the edge of trade wind cumuli: Observations and dynamical implications. Atmos. Res., 97, 144-156, doi:10.1016/ j.atmosres.2010.03.017.

,-- , and J. French, 2009: Dynamics of the cumulus cloud margin: An observational study. J. Atmos. Sci., 66, 3660-3677, doi:10.1175/2009JAS3129.1.

Woodward, B., 1959: The motion in and around isolated thermals. Quart. J. Roy. Meteor. Soc., 85, 144-151, doi:10.1002/ qj.49708536407.

Wyngaard, J. C., 2010: Turbulence in the Atmosphere. Cambridge University Press, 408 pp.

Zhao, M., and P. H. Austin, 2005a: Life cycle of numerically simulated shallow cumulus clouds. Part I: Transport. J. Atmos. Sci., 62, 1269-1290, doi:10.1175/JAS3414.1.

— and -2005 b: Life cycle of numerically simulated shallow cumulus clouds. Part II: Mixing dynamics. J. Atmos. Sci., 62, 1291-1310, doi:10.1175/JAS3415.1. 